\title{
Proportion of Aspiration Pneumonia Cases Among Patients With Community-Acquired Pneumonia: A Single-Center Study in Korea
}

\author{
Inpyo Jeon, $\mathrm{MD}^{1}$, Gwang Pyo Jung, $\mathrm{MD}^{1}$, Han Gil Seo, MD, $\mathrm{PhD}^{2}$, \\ Ju Seok Ryu, MD, $\mathrm{PhD}^{1,3}$, Tai Ryoon Han, $\mathrm{MD}, \mathrm{PhD}^{1}$, Byung-Mo Oh, MD, $\mathrm{PhD}^{1,2}$ \\ ${ }^{1}$ Department of Rehabilitation Medicine, Seoul National University College of Medicine, Seoul; \\ ${ }^{2}$ Department of Rehabilitation Medicine, Seoul National University Hospital, Seoul; \\ ${ }^{3}$ Department of Rehabilitation Medicine, Seoul National University Bundang Hospital, Seongnam, Korea
}

\begin{abstract}
Objective To investigate the proportion of aspiration pneumonia cases among patients with community-acquired pneumonia in Korea.

Methods This retrospective study included patients with community-acquired pneumonia who had been admitted to the emergency department of a university-affiliated tertiary hospital in Gyeonggi Province, Korea between January 1, 2016 and December 31, 2016. Among these patients, those with aspiration pneumonia were identified using ICD-10 codes (J69.*). Patients with recurrent pneumonia were excluded, as were those who were immunocompromised. The proportion of cases of aspiration pneumonia was calculated, and the characteristics and clinical outcomes of patients with aspiration pneumonia and non-aspiration pneumonia were compared.

Results The proportion of aspiration pneumonia cases among patients with community-acquired pneumonia was $14.2 \%$. Patients with aspiration pneumonia were significantly more likely to be older $(\mathrm{p}<0.001)$ and male $(\mathrm{p}<0.001)$, and to have a higher confusion, uremia, respiratory rate, blood pressure, and age $\geq 65$ years (CURB-65) score $(\mathrm{p}<0.001)$ as compared to patients with non-aspiration pneumonia. They were also more likely to require admission to the intensive care unit $(\mathrm{p}<0.001)$ and a longer hospital stay $(\mathrm{p}<0.001)$.

Conclusion Aspiration pneumonia accounts for $14.2 \%$ of all cases of community-acquired pneumonia in Korea. These data may contribute to the establishment of healthcare strategies for managing aspiration pneumonia among Korean adults.
\end{abstract}

Keywords Aspiration pneumonia, Community-acquired infections, Proportion

Received July 19, 2018; Accepted October 10, 2018

Corresponding author: Byung-Mo Oh

Department of Rehabilitation Medicine, Seoul National University Hospital, 101 Daehak-ro, Jongno-gu, Seoul 03080, Korea. Tel: +82-2-2072-2619, Fax: +82-2-743-7473, E-mail: moya1@snu.ac.kr

ORCID: Inpyo Jeon (http://orcid.org/0000-0001-5576-0435); Gwang Pyo Jung (http://orcid.org/0000-0002-0938-1546); Han Gil Seo (http://orcid. org/0000-0001-6904-7542); Ju Seok Ryu (http://orcid.org/0000-0003-3299-3038); Tai Ryoon Han (http://orcid.org/0000-0002-7532-9068); Byung-Mo Oh (http://orcid.org/0000-0001-9353-7541).

(c) This is an open-access article distributed under the terms of the Creative Commons Attribution Non-Commercial License (http://creativecommons.org/ licenses/by-nc/4.0) which permits unrestricted noncommercial use, distribution, and reproduction in any medium, provided the original work is properly cited. Copyright ( 2019 by Korean Academy of Rehabilitation Medicine 


\section{INTRODUCTION}

Pneumonia is the second-leading cause of death worldwide [1] and the fourth-leading cause of death in Korea [2]. Between 2011 and 2014 in Korea, the overall incidence of community-acquired pneumonia was 307.3 cases per 100,000 people per year [3]. Further, the mortality rate increased 3.5 -fold over a 10 -year span, from 9.3 deaths per 100,000 people in 2006 to 32.2 per 100,000 people in 2016 [2]. Both the number of patients hospitalized for pneumonia and the associated medical costs increased between 2012 and 2016; the number of patients increased by $26.2 \%$ (from 320,000 to 410,000 ) while medical costs increased by $50.1 \%$ (from 483 billion Korean won to 718.8 billion Korean won) [4].

Aspiration pneumonia is a distinct subtype of pneumonia [5]. Specifically, it is an acute bacterial pneumonia that occurs as a result of macro-aspiration of oropharyngeal or upper gastrointestinal contents [6]. Aspiration pneumonia is more severe than non-aspiration pneumonia [5], with higher rates of mortality and recurrence as well as increased lengths of hospital stay as compared to non-aspiration pneumonia [5]. Although aspiration pneumonia is known to significantly contribute to pneumonia-associated deaths, its incidence has yet to be fully elucidated [5,7-9].

Older adults are at higher risk of aspiration pneumonia [10]; thus, it is unsurprising that this same group exhibits a higher incidence of aspiration pneumonia [11]. Korea is one of the fastest aging societies, in which the number of adults aged 65 years and older will increase dramatically to $37.3 \%$ by 2050 [12]. Therefore, aspiration pneumonia will become an even more important public health issue in the future and will thus require a greater investment of resources for disease management. However, there are currently no epidemiological data available regarding community-acquired aspiration pneumonia in Korea.

Diagnosing pneumonia in older adults can be difficult [13]. Chest radiography is essential for confirming the diagnosis [13], while chest computed tomography (CT) is a valuable adjunct $[14,15]$. This is because chest CT may provide valuable diagnostic information in bedridden or elderly patients, because the presence of kyphosis, muscular weakness, and neurological disorder limits the ability to obtain accurate X-ray images in these patients [13]. Aspiration pneumonia is known as gravity- dependent pneumonia [16]; its radiographic appearance varies based on a number of factors, but it commonly involves the dependent (posterior) portion bilaterally [1416]. Chest CT is better than plain chest radiography in terms of localizing the pulmonary infiltrates of aspiration pneumonia. Despite its overall value as a diagnostic tool, no studies have directly assessed the value of chest CT in diagnosing aspiration pneumonia.

In addition to CT, videofluoroscopic swallowing study (VFSS) is a well-known diagnostic tool for oropharyngeal aspiration [17], which is an etiologic agent of aspiration pneumonia $[6,18]$. However, there have been no studies evaluating the value of VFSS as a diagnostic tool for aspiration pneumonia.

Therefore, the aims of this study were to: (1) estimate the proportion of aspiration pneumonia cases among patients with community-acquired pneumonia in Korea; (2) compare the characteristics and outcomes of patients with community-acquired aspiration pneumonia to those of patients with community-acquired non-aspiration pneumonia; and (3) evaluate the diagnostic value of chest CT and VFSS for community-acquired aspiration pneumonia.

\section{MATERIALS AND METHODS}

\section{Study design and data source}

The clinical data for this retrospective study were obtained from the electronic medical records of Seoul National University Bundang Hospital, a 1,432-bed urban teaching hospital in Korea; this hospital is one of seven regional emergency centers in Gyeonggi-do [19], a province with a population of 12,716,780 as of 2016 [20]. The Institutional Review Board at Seoul National University Bundang Hospital approved this study (No. B-1808-484114). All medical records were fully anonymized prior to analysis. The requirement for written informed consent was waived because all data were collected retrospectively through the review of medical records.

\section{Selection of study population}

In this study, we analyzed data from patients aged 18 years and older who had been to the emergency department between January 1, 2016 and December 31, 2016 with a diagnosis of pneumonia (ICD-10 codes A24.1, A50.0, A54.8, B01.2, B05.2, B06.8, B59, B96.0, J10.0, J11.0, 
J12.*, J13, J14, J15.*, J16.*, J17.1, J17.3, J17.8, J18.*, J20.0) [4] and/or aspiration pneumonia (ICD-10 code J69.*). Exclusion criteria included the following: (1) recurrent pneumonia; (2) hospital-acquired pneumonia; (3) HIV/AIDS infection; (4) lung cancer; (5) solid organ transplantation; and/or (6) hematologic malignancy. Hospital-acquired pneumonia was defined as pneumonia that had not incubated at the time of hospital admission and occurred 48 hours or more after admission [21]. The definition of community-acquired pneumonia included healthcareassociated pneumonia [21]. We classified communityacquired pneumonia as aspiration or non-aspiration pneumonia using the primary diagnosis code.

\section{Clinical characteristics}

Data regarding the general and clinical characteristics as well as the type of diagnostic tests were acquired for all patients with pneumonia. The general characteristics included sex and age, while the clinical characteristics included underlying disease; confusion, uremia, respiratory rate, blood pressure, age $\geq 65$ years (CURB-65) score [22]; intensive care unit (ICU) admission; and length of hospital stay. CURB-65 is a community-acquired pneumonia severity score based on confusion, urea ( $>7 \mathrm{mmol} /$ L), respiratory rate ( $\geq 30$ breaths $/ \mathrm{min}$ ), blood pressure (systolic blood pressure $<90 \mathrm{mmHg}$ or diastolic blood pressure $\leq 60 \mathrm{mmHg}$ ), and age ( $\geq 65$ years). Chest $\mathrm{CT}$ and
VFSS were performed in order to calculate the proportion of aspiration pneumonia cases with objective evidence of aspiration and to assess their value as diagnostic tests in terms of sensitivity, specificity, predictive value, and likelihood ratio.

\section{Statistical analysis}

Descriptive statistics were used to summarize the data. The proportion of aspiration pneumonia cases was calculated. We compared the characteristics of cases of aspiration and non-aspiration pneumonia using chisquared and independent t-tests. p-values below 0.05 were considered to be statistically significant. All data were analyzed using IBM SPSS Statistics version 23.0 (IBM, Chicago, IL, USA).

\section{RESULTS}

Data from a total of 1,597 patients were considered for analysis. Four hundred thirty-five patients had hospitalacquired pneumonia and 117 patients had lung cancer, hematologic malignancy, or a history of solid organ transplantation, and were thus excluded. No patients were infected with HIV/AIDS. Three patients without any admission notes were also excluded. In total, 1,042 patients met the inclusion criteria for analysis; all of these had been diagnosed with community-acquired pneumonia (Fig. 1).

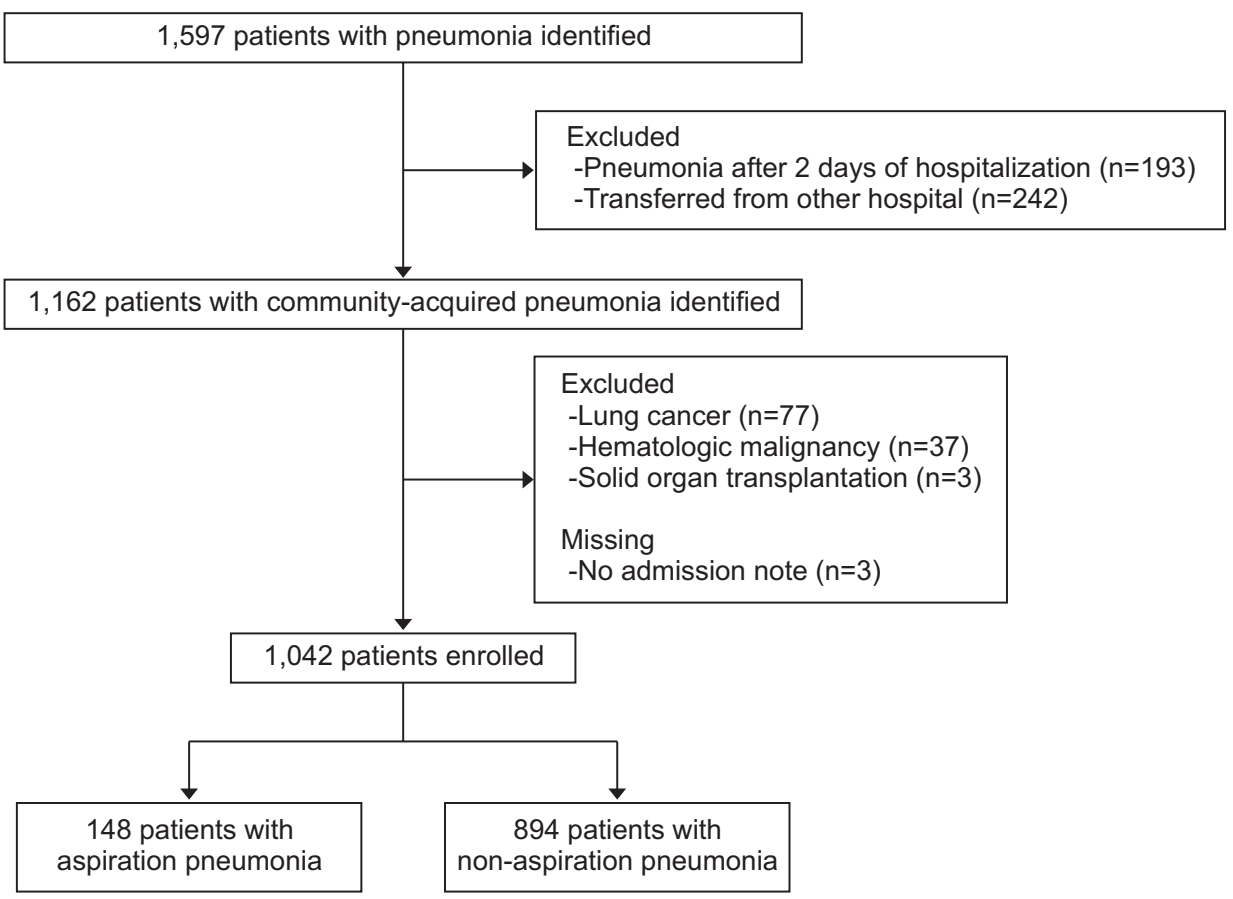

Fig. 1. Flowchart of study subjects. 
Among the patients with community-acquired pneumonia, 148 were diagnosed with aspiration pneumonia. In this study, the proportion of aspiration pneumonia cases among patients with community-acquired pneumonia was $14.2 \%$ (Table 1 ).

As compared to patients with non-aspiration pneumonia, those with aspiration pneumonia were significantly older $(\mathrm{p}<0.001)$ and more likely to be male $(\mathrm{p}<0.001)$. These patients required significantly longer hospital stays $(\mathrm{p}<0.001)$ and more frequent ICU admissions $(\mathrm{p}<0.001)$, and also had higher CURB-65 scores $(\mathrm{p}<0.001)$ (Table 1$)$.

Among the 1,042 patients with community-acquired pneumonia, $623(59.8 \%)$ were examined by chest CT. One hundred and thirteen of the patients had aspiration pneumonia as confirmed by a positive chest CT scan. Therefore, based on the chest CT results, $18.1 \%$ of the community-acquired pneumonia patients had aspiration pneumonia. The sensitivity and specificity values of chest CT were $70.8 \%$ and $93.5 \%$, respectively, and the positive and negative predictive values of chest CT were $70.8 \%$ and $93.5 \%$, respectively. In this study, the positive likelihood ratio was 10.1 while the negative likelihood ratio was 0.312 (Table 2).

Among the 1,042 patients with community-acquired pneumonia, 60 (5.8\%) completed a VFSS. The sensitivity and specificity values of VFSS were $62.5 \%$ and $100 \%$, respectively; while the positive and negative predictive values of VFSS were $100 \%$ and $70 \%$, respectively. In this study, the positive likelihood ratio was undefined and the negative likelihood ratio was 0.375 (Table 2).

\section{DISCUSSION}

This is the first study to estimate the proportion of aspiration pneumonia cases among patients with community-acquired pneumonia in Korea. This was also the first study to examine the values of chest CT and VFSS as diagnostic tools for aspiration pneumonia.

Table 2. Chest CT and VFSS findings

\begin{tabular}{lcc}
\hline & \multicolumn{2}{c}{$\begin{array}{c}\text { Findings supporting } \\
\text { diagnosis }\end{array}$} \\
\cline { 2 - 3 } & Positive & Negative \\
\hline Chest CT (n=623) & & \\
Aspiration pneumonia $(\mathrm{n}=113)$ & 80 & 33 \\
$\begin{array}{l}\text { Non-aspiration pneumonia } \\
\quad(\mathrm{n}=510)\end{array}$ & 33 & 477 \\
$\begin{array}{l}\text { VFSS (n=60) } \\
\text { Aspiration pneumonia (n=32) }\end{array}$ & 20 & 12 \\
$\begin{array}{l}\text { Non-aspiration pneumonia } \\
(\mathrm{n}=28)\end{array}$ & 0 & 28 \\
\hline
\end{tabular}

CT, computed tomography; VFSS, videofluoroscopic swallowing study.

Table 1. Characteristics of patients with aspiration and non-aspiration pneumonia

\begin{tabular}{|lcccr|}
\hline & Total & $\begin{array}{c}\text { Aspiration } \\
\text { pneumonia }\end{array}$ & $\begin{array}{c}\text { Non-aspiration } \\
\text { pneumonia }\end{array}$ & p-value \\
\hline Number of patients & $1,042(100)$ & $148(14.2)$ & $894(85.8)$ & - \\
\hline Age (yr) & $65.3 \pm 18.8$ & $73.8 \pm 16.7$ & $63.9 \pm 18.7$ & $<0.001$ \\
\hline Sex, male & $572(54.9)$ & $102(68.9)$ & $470(54.6)$ & $<0.001$ \\
\hline Intensive care unit admission & $127(12.2)$ & $39(26.3)$ & $88(9.8)$ & $<0.001$ \\
\hline CURB-65 score ${ }^{\text {a) }}$ & $1(0-2)$ & $3(2-3)$ & $1(0-2)$ & $<0.001$ \\
\hline Confusion & $65(7.3)$ & $15(33.3)$ & $50(5.9)$ & $<0.001$ \\
\hline Urea (mmol/L) & $15(10-22)$ & $28(21-37)$ & $14(10-21)$ & $<0.001$ \\
\hline Respiratory rate (breaths/min) & $19(18-22)$ & $22(18-24)$ & $19(18-22)$ & 0.011 \\
\hline Blood pressure (mmHg) & $130(115-149)$ & $117(102-135)$ & $131(116-149)$ & 0.007 \\
\hline Length of stay (day) & $3(1-10)$ & $9(2-15)$ & $2(1-9)$ & $<0.001$ \\
\hline
\end{tabular}

Values are presented as number (\%) or mean \pm standard deviation or median (interquartile range, 25th-75th percentile).

${ }^{\text {a) }}$ CURB-65 is a community-acquired pneumonia severity score based on confusion, urea $(>7 \mathrm{mmol} / \mathrm{L})$, respiratory rate $(\geq 30 / \mathrm{min}$ ), blood pressure (systolic blood pressure $<90 \mathrm{mmHg}$ or diastolic blood pressure $\leq 60 \mathrm{mmHg}$ ), and age $(\geq 65$ years). The scores were defined as 'low' for CURB-65 total scores of 0-1 point, as 'moderate' for scores of 2 points, and as 'high' for scores of 3-5 points. 
In the present study, among patients with communityacquired pneumonia, $14.2 \%$ were diagnosed with aspiration pneumonia. Previous studies have reported the proportions of cases of aspiration pneumonia to range from $5 \%$ to $60 \%[5,7-9,23]$. Among these studies, only one estimated the proportion of aspiration pneumonia cases using distinct diagnostic codes related to aspiration pneumonia; that study found a proportion of aspiration pneumonia cases of $16.5 \%$ over a 10 -year span [8], which was consistent with our findings.

The risk of aspiration pneumonia increases in older adults [9-11], which is particularly relevant in the rapidly aging society of Korea [4]. Therefore, as the incidence of aspiration pneumonia may increase in Korea, urgent preventive measures are required. Progressive obtundation of the swallowing reflex with age is likely to be one of the major causes of the high incidence of aspiration pneumonia among older adults [24]. Various efforts have gone toward mitigating the pathologic process of agerelated coughing and swallowing difficulty [25]. Because dysphagia and its resulting repeated silent aspiration is an important mechanism in the pathogenesis of aspiration pneumonia in older people $[9,25]$, screening for and early intervention in dysphagia may become an important public health issue. Approximately half of nursing home residents in Korea have dysphagia [26]; however, VFSS is not commonly used to test pneumonia patients. Specifically, a previous study reported that, in Japan, only $6.2 \%$ of patients with pneumonia and $5.8 \%$ of patients with community-acquired pneumonia in particular were examined using VFSS [9]. Therefore, a strategy for preventing aspiration pneumonia should include additional efforts to perform VFSS examinations.

In this study, we used ICD-10 codes related to aspiration pneumonia (J69.*) in order to differentiate aspiration from non-aspiration pneumonia. As there is no gold standard for defining aspiration pneumonia, previous research has been limited to definitions created by the investigators [8]. In addition to the use of rarely-witnessed aspiration events [27], some reports have included diagnoses of aspiration pneumonia based on aspiration risk factors, such as cerebrovascular disease or dementia $[5,28,29]$; other reports used chest CT, rather than chest $\mathrm{X}$-ray imaging, to identify radiograph infiltrates in dependent pulmonary segments $[30,31]$. Another study used swallowing function tests, including VFSS findings, to determine aspiration tendency [9]. Instead of using an evidence-based diagnosis [29] for aspiration pneumonia (as the present study did), some previous studies have employed clinical definitions of the presence of aspiration pneumonia, such as clinicians' reports [8], physician billing codes [23], or diagnosis codes [32]. However, in practice, a clinician's means of diagnosing a patient with aspiration pneumonia are likely to be vague and inconsistent [8]. Due to the lack of a sensitive and specific marker for aspiration, along with the potential overlap between aspiration pneumonia and other forms of pneumonia, clinicians often experience difficulty diagnosing aspiration pneumonia [8]. However, the strength of the 'wisdom of the crowd' method may reduce the noise from individual judgment and help provide reliable results [8]. In this study, our findings regarding aspiration pneumonia characteristics, including the observation of more severe outcomes than those seen in non-aspiration pneumonia, are similar to those from studies using investigator-based definitions [5,31].

The proportions of aspiration pneumonia cases reported in previous studies range from 5-60\% [5,7-9,23]. For example, Marik [7] reported a proportion of 5\%-15\% based on three studies published before 2001. Further, based on a single tertiary university-affiliated hospital study in the United States, Lanspa et al. [23] reported that between 1996 and 2006, the proportion of aspiration pneumonia cases among patients with community-acquired pneumonia was $16.5 \%$. By analyzing multicenter and multinational data, they later reported that, between 2001 and 2012, 8.7\% of patients with communityacquired pneumonia exhibited aspiration pneumonia [8]. In addition, Hayashi et al. [5] reported that, between 2010 and 2012, aspiration pneumonia was diagnosed in $33.6 \%$ of patients with community-acquired or healthcareassociated pneumonia in Japan. Another Japanese study conducted by Teramoto et al. [9] reported that $60 \%$ of patients with community-acquired pneumonia exhibited aspiration pneumonia. These discrepancies in the findings among studies may arise from differences in the case definitions of aspiration pneumonia [3]. In 2013, Lanspa et al. [23] used diagnostic codes to define aspiration pneumonia; they found a proportion of cases of $16.5 \%$, which was similar to ours (14.2\%). In our study, among patients with community-acquired pneumonia who were examined using chest CT, $12.8 \%$ were diagnosed with as- 
piration pneumonia.

This study also found that, as compared to patients with non-aspiration pneumonia, patients with aspiration pneumonia were more likely to be older, male, and exhibit a severe disease state requiring a longer hospital stay. These characteristics are similar to those described in previous studies, which reported that patients with aspiration pneumonia were typically older and male $[8,11]$ and exhibited greater disease severity and more comorbidities $[8,23]$. Aspiration pneumonia patients were also admitted to ICUs more frequently and had longer hospital stays [8].

Male patients were significantly more susceptible to aspiration pneumonia than other types of communityacquired pneumonia. Previous studies have reported that the risk of dysphagia does not differ significantly between males and females [33,34]. Therefore, the immune response to oropharyngeal aspirates may differ between sexes, and this difference may be attributed to estrogens and progestins [35].

When VFSS was used as an additional diagnostic test for aspiration pneumonia among patients with communityacquired pneumonia, it was found to have $100 \%$ specificity and a positive predictive value. This diagnostic test accuracy may be affected by incorporation bias and an imperfect gold standard [36]; incorporation bias occurs when the classification of disease status partly depends on the results of the index test, while imperfect gold standard bias occurs when the standard determining a patient's true disease status misclassifies certain patients. Despite these biases, VFSS may be an important diagnostic test for aspiration pneumonia, and as such should be incorporated more frequently into the diagnostic process for patients with potential aspiration pneumonia.

Bedside swallowing screening tests are recommended for patients at high risk of oropharyngeal aspiration, such as acute stroke patients. For patients with abnormal screening tests results, brainstem lesion, or multifocal infarction, VFSS or fiberoptic endoscopic evaluation of swallowing is recommended [37]. A previous study suggested that elderly patients with clinical signs of dysphagia and/or those with community-acquired pneumonia should be referred for a swallowing evaluation [18]. Therefore, in line with the clinical practice guidelines for stroke patients in Korea [37], bedside swallowing screening tests should be recommended for patients with acute community-acquired pneumonia. VFSS should also be recommended for patients with community-acquired pneumonia who exhibit abnormal bedside swallowing screening tests and/or those with aspiration pneumonia.

This study had several limitations. First, this was a single hospital-based study; therefore, our findings may not be generalizable to all of Korea. However, the results of our study are consistent with the previously reported findings of studies that used similar designs. We used clinical determination for the diagnosis of aspiration pneumonia. We also used the findings reported by various physicians for chest CT and VFSS examinations. However, because this study was conducted at a single university-affiliated hospital, the methods used for interpreting the test results may have been relatively homogeneous. Lastly, patient comorbidities were not analyzed. This is a limitation because comorbidities (e.g., cerebrovascular disease, degenerative diseases, and dementia) increase the risk of aspiration [5]. The hospital in this study was a university-affiliated tertiary hospital; therefore, the admitted pneumonia patients may have exhibited more comorbidities than patients in typical community hospitals, which may have resulted in an overestimation of the proportion of aspiration pneumonia cases among patients with community-acquired pneumonia.

Despite these limitations, this study is the first to estimate the proportion of aspiration pneumonia in Korea, and may therefore constitute the first step in estimating the national proportion of community-acquired aspiration pneumonia cases.

In this study, the proportion of aspiration pneumonia cases among patients with community-acquired pneumonia was found to be $14.2 \%$ in 2016 . Considering that the incidence of community-acquired pneumonia has been reported to be 307.3 cases per 100,000 people per year [3], the estimated incidence of community-acquired aspiration pneumonia may be as high as 43.6 cases per 100,000 people per year. We found that the VFSS was highly sensitive for diagnosis; however, only $5.8 \%$ of patients with community-acquired pneumonia were examined by VFSS. In the future, VFSS should be considered an important diagnostic method. Further, additional studies should be performed in order to estimate the national proportion of community-acquired aspiration pneumonia. This study thus serves as the first step in establishing healthcare strategies and estimating the im- 
pact of aspiration pneumonia among Korean adults.

\section{CONFLICT OF INTEREST}

No potential conflict of interest relevant to this article was reported.

\section{AUTHOR CONTRIBUTION}

Conceptualization: Jeon I, Seo HG, Han TR, Oh BM. Methodology: Jeon I, Jung GP, Seo HG, Ryu JS, Oh BM. Formal analysis: Jeon I, Oh BM. Funding acquisition: none. Project administration: Jeon I, Jung GP, Oh BM. Visualization: Jeon I, Oh BM. Writing-original draft: Jeon I, Oh BM. Writing-review and editing: Jeon I, Jung GP, Seo HG, Ryu JS, Oh BM. Approval of final manuscript: all authors.

\section{REFERENCES}

1. Mokdad AH, Forouzanfar MH, Daoud F, Mokdad AA, El Bcheraoui C, Moradi-Lakeh M, et al. Global burden of diseases, injuries, and risk factors for young people's health during 1990-2013: a systematic analysis for the Global Burden of Disease Study 2013. Lancet 2016;387:2383-401.

2. Korean Statistical Information Service. Vital Statistics 2016 [Internet]. Daejeon: Statistics Korea; c2018 [cited 2019 Apr 1]. Available from: http://kosis.kr/statHtml/statHtml.do? orgId=101\&tblId=DT_1B34E07\&vw_cd=MT_ZTITLE\&list_ id $=$ D11\&seqNo $=\& l a n g \_$mode $=$ko\&language $=$kor\&obj var_id=\&itm_id=\&conn_path=MT_ZTITLE\#.

3. Heo JY, Seo YB, Choi WS, Lee J, Yoon JG, Lee SN, et al. Incidence and case fatality rates of communityacquired pneumonia and pneumococcal diseases among Korean adults: catchment population-based analysis. PLoS One 2018;13:e0194598.

4. Health Insurance Review \& Assessment Service. 2016 National pneumonia treatment propriety assessment program. Wonju: Health Insurance Review \& Assessment Service; 2017.

5. Hayashi M, Iwasaki T, Yamazaki Y, Takayasu H, Tateno $\mathrm{H}$, Tazawa S, et al. Clinical features and outcomes of aspiration pneumonia compared with non-aspiration pneumonia: a retrospective cohort study. J Infect Chemother 2014;20:436-42.
6. DiBardino DM, Wunderink RG. Aspiration pneumonia: a review of modern trends. J Crit Care 2015;30:408.

7. Marik PE. Aspiration pneumonitis and aspiration pneumonia. N Engl J Med 2001;344:665-71.

8. Lanspa MJ, Peyrani P, Wiemken T, Wilson EL, Ramirez JA, Dean NC. Characteristics associated with clinician diagnosis of aspiration pneumonia: a descriptive study of afflicted patients and their outcomes. J Hosp Med 2015;10:90-6.

9. Teramoto S, Fukuchi Y, Sasaki H, Sato K, Sekizawa K, Matsuse T, et al. High incidence of aspiration pneumonia in community- and hospital-acquired pneumonia in hospitalized patients: a multicenter, prospective study in Japan. J Am Geriatr Soc 2008;56:5779.

10. Loeb MB, Becker M, Eady A, Walker-Dilks C. Interventions to prevent aspiration pneumonia in older adults: a systematic review. J Am Geriatr Soc 2003;51:1018-22.

11. Palacios-Cena D, Hernandez-Barrera V, Lopez-deAndres A, Fernandez-de-Las-Penas C, Palacios-Cena M, de Miguel-Diez J, et al. Time trends in incidence and outcomes of hospitalizations for aspiration pneumonia among elderly people in Spain (2003-2013). Eur J Intern Med 2017;38:61-7.

12. Kim SH, Kim DH, Kim WS. Long-term care needs of the elderly in Korea and elderly long-term care insurance. Soc Work Public Health 2010;25:176-84.

13. Esayag Y, Nikitin I, Bar-Ziv J, Cytter R, Hadas-Halpern I, Zalut T, et al. Diagnostic value of chest radiographs in bedridden patients suspected of having pneumonia. Am J Med 2010;123:88.e1-5.

14. Franquet T. Imaging of pneumonia: trends and algorithms. Eur Respir J 2001;18:196-208.

15. Sharma S, Maycher B, Eschun G. Radiological imaging in pneumonia: recent innovations. Curr Opin Pulm Med 2007;13:159-69.

16. Komiya K, Ishii H, Umeki K, Kawamura T, Okada F, Okabe E, et al. Computed tomography findings of aspiration pneumonia in 53 patients. Geriatr Gerontol Int 2013;13:580-5.

17. Oh BM, Lee JH, Seo HG, Lee WH, Han TR, Jeong SU, et al. Changes in hyolaryngeal movement during swallowing in the lateral decubitus posture. Ann Rehabil Med 2018;42:416-24.

18. Marik PE, Kaplan D. Aspiration pneumonia and dys- 
phagia in the elderly. Chest 2003;124:328-36.

19. National Emergency Medical Center. 2016 Emergency medical institution status [Internet]. Seoul: National Emergency Medical Center; c2019 [cited 2019 Apr 1]. Available from: http://www.e-gen.or.kr/nemc/notice_ view.do?brdctsno $=1753 \&$ upperfixyn $=N \&$ currentPage Num $=1 \&$ brdclscd $=01 \&$ searchTarget=ALL\&searchKey

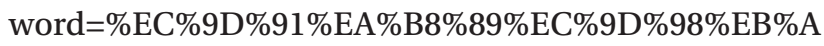
3\%8С\%ЕА\%В8\%В0\%ЕА\%В4\%80\%20\%ED\%98\%84\%ED \%99\%A9\&searchDatayear $=$.

20. Korean Statistical Information Service. Population Census [Internet]. Daejeon: Statistics Korea; c2019 [cited 2019 Apr 1]. Available from: http://kosis.kr/ statHtml/statHtml.do?orgId=101\&tblId=DT_1B0 $40 \mathrm{~A} 3 \& v w_{-}$cd $=\& l i s t \_i d=\&$ scrId $=\&$ seqNo $=\& l a n g$ mode $=$ ko\&obj_var_id=\&itm_id=\&conn_path=El\#.

21. American Thoracic Society; Infectious Diseases Society of America. Guidelines for the management of adults with hospital-acquired, ventilator-associated, and healthcare-associated pneumonia. Am J Respir Crit Care Med 2005;171:388-416.

22. Yoo KH, Yoo CG, Kim SK, Jung JY, Lee MG, Uh ST, et al. Economic burden and epidemiology of pneumonia in Korean adults aged over 50 years. J Korean Med Sci 2013;28:888-95.

23. Lanspa MJ, Jones BE, Brown SM, Dean NC. Mortality, morbidity, and disease severity of patients with aspiration pneumonia. J Hosp Med 2013;8:83-90.

24. Ebihara S, Ebihara T, Kohzuki M. Effect of aging on cough and swallowing reflexes: implications for preventing aspiration pneumonia. Lung 2012;190:29-33.

25. Ebihara S, Sekiya H, Miyagi M, Ebihara T, Okazaki T. Dysphagia, dystussia, and aspiration pneumonia in elderly people. J Thorac Dis 2016;8:632-9.

26. Park YH, Han HR, Oh BM, Lee J, Park JA, Yu SJ, et al. Prevalence and associated factors of dysphagia in nursing home residents. Geriatr Nurs 2013;34:212-7.

27. Bartlett JG, Gorbach SL. The triple threat of aspiration pneumonia. Chest 1975;68:560-6.
28. Mier L, Dreyfuss D, Darchy B, Lanore JJ, Djedaini K, Weber $P$, et al. Is penicillin $G$ an adequate initial treatment for aspiration pneumonia? A prospective evaluation using a protected specimen brush and quantitative cultures. Intensive Care Med 1993;19:279-84.

29. Sue Eisenstadt E. Dysphagia and aspiration pneumonia in older adults. J Am Acad Nurse Pract 2010;22:1722.

30. Tokuyasu H, Harada T, Watanabe E, Okazaki R, Touge $\mathrm{H}$, Kawasaki Y, et al. Effectiveness of meropenem for the treatment of aspiration pneumonia in elderly patients. Intern Med 2009;48:129-35.

31. Komiya K, Ishii H, Umeki K, Mizunoe S, Okada F, Johkoh $\mathrm{T}$, et al. Impact of aspiration pneumonia in patients with community-acquired pneumonia and healthcare-associated pneumonia: a multicenter retrospective cohort study. Respirology 2013;18:514-21.

32. Wu CP, Chen YW, Wang MJ, Pinelis E. National trends in admission for aspiration pneumonia in the United States, 2002-2012. Ann Am Thorac Soc 2017;14:874-9.

33. Byeon H. Analysis of dysphagia risk using the modified dysphagia risk assessment for the communitydwelling elderly. J Phys Ther Sci 2016;28:2507-9.

34. Cho SY, Choung RS, Saito YA, Schleck CD, Zinsmeister AR, Locke GR 3rd, et al. Prevalence and risk factors for dysphagia: a USA community study. Neurogastroenterol Motil 2015;27:212-9.

35. Kwon HM, Jeong SW, Lee SH, Yoon BW. The pneumonia score: a simple grading scale for prediction of pneumonia after acute stroke. Am J Infect Control 2006;34:64-8.

36. Kohn MA, Carpenter CR, Newman TB. Understanding the direction of bias in studies of diagnostic test accuracy. Acad Emerg Med 2013;20:1194-206.

37. Kim DY, Kim YH, Lee J, Chang WH, Kim MW, Pyun SB, et al. Clinical practice guideline for stroke rehabilitation in Korea 2016. Brain Neurorehabil 2017;10(Suppl 1):e11. 